\title{
Reconstruction of Surfaces of Revolution from Single Uncalibrated Views
}

\author{
Kwan-Yee K. Wong* \\ Department of Computer Science \& Information Systems \\ The University of Hong Kong, Pokfulam Rd, HK \\ kykwong@CCsis.hku.hk \\ Paulo R. S. Mendonça \\ GE Global Research Center \\ Schenectady, NY 12301, USA \\ mendonca@crd.ge.com \\ Roberto Cipolla \\ Department of Engineering, University of Cambridge \\ Trumpington Street, Cambridge CB2 1PZ, UK \\ cipolla@eng.cam.ac.uk
}

\begin{abstract}
This paper addresses the problem of recovering the 3D shape of a surface of revolution from a single uncalibrated perspective view. The algorithm introduced here makes use of the invariant properties of a surface of revolution and its silhouette to locate the image of the revolution axis, and to calibrate the focal length of the camera. The image is then normalized and rectified such that the resulting silhouette exhibits bilateral symmetry. Such a rectification leads to a simpler differential analysis of the silhouette, and yields a simple equation for depth recovery. Ambiguities in the reconstruction are analyzed and experimental results on real images are presented, which demonstrate the quality of the reconstruction.
\end{abstract}

\section{Introduction}

2D images contain cues to surface shape and orientation. However, their interpretation is inherently ambiguous because depth information is lost during the image formation process when 3D structures in the world are projected onto 2D images. Multiple images from different viewpoints can be used to resolve these ambiguities, and this results in techniques like stereo vision $[8,1]$ and structure from motion $[16,11]$. Besides, under certain appropriate assumptions such as Lambertian surfaces and isotropic textures, it is also possible to infer scene structure (e.g. surface orientation and curvature) from a single image using techniques like shape from shading [21] and shape from texture [12]. In fact,

\footnotetext{
${ }^{*}$ This project is partially funded by The University of Hong Kong.
} 
if some strong a priori knowledge of the object is available, such as the class of shapes to which the object belongs, then a single view alone allows shape recovery. Examples of such techniques can be found in $[7,14,10,15,6,20,17,19]$, where the invariant and quasi-invariant properties of some generalized cylinders (GCs) [2] and their silhouettes were exploited to derive algorithms for segmentation and 3D recovery of the GCs under orthographic projection.

This paper addresses the problem of recovering the 3D shape of a surface of revolution (SOR) from a single view. Surfaces of revolution belong to a subclass of straight homogeneous GCs, in which the planar cross-section is a circle centered at and orthogonal to its axis. This work is different from the previous ones in that, rather than the orthographic projection model, which is a quite restricted case, the perspective projection model is assumed. In [9], Lavest et al. presented a system for modelling SORs from a set of few monocular images. Their method requires a perspective image of an "angular ridge" of the object to determine the attitude of the object, and it only works with calibrated cameras. The algorithm introduced here works with an uncalibrated camera, and it estimates the focal length of the camera directly from the silhouette. Besides, an "angular ridge" is not necessary as the algorithm produces a 2-parameter family of SORs under an unknown attitude and scale of the object.

This paper is organized as follows. Section 2 gives the theoretical background necessary for the development of the algorithm presented in this paper. A parameterization for surfaces of revolution is presented and the symmetry properties exhibited in the silhouettes are summarized. In particular, the surface normal and the revolution axis are shown to be coplanar. This coplanarity constraint is exploited in Section 3 to derive a simple technique for reconstructing a surface of revolution from its silhouette in a single view. It is shown that under a general camera configuration, there will be a 2-parameter family of solutions for the reconstruction. The first parameter corresponds to an unknown scale in the reconstruction resulting from the unknown distance of the surface from the camera. The second parameter corresponds to the ambiguity in the orientation of the revolution axis on the $y-z$ plane of the camera coordinate system. It is shown in the Appendix that such ambiguities in the reconstruction cannot be described by a projective transformation. The algorithm and implementation are described in Section 4 and results of real data experiments are presented in Section 5. Finally conclusions are given in Section 6.

\section{Properties of Surfaces of Revolution}

Let $\tilde{\mathbf{C}}_{\mathrm{r}}(s)=\left[\begin{array}{lll}X(s) & Y(s) & 0\end{array}\right]^{\mathrm{T}}$ be a regular and differentiable planar curve on the $x-y$ plane where $X(s)>0$ for all $s$. A surface of revolution can be generated by rotating $\tilde{\mathbf{C}}_{\mathrm{r}}$ about the $y$-axis, and is given by

$$
\tilde{\mathbf{S}}_{\mathrm{r}}(s, \theta)=\left[\begin{array}{c}
X(s) \cos \theta \\
Y(s) \\
X(s) \sin \theta
\end{array}\right],
$$

where $\theta$ is the angle parameter for a complete circle. The tangent plane basis vectors

$$
\frac{\partial \tilde{\mathbf{S}}_{\mathrm{r}}}{\partial s}=\left[\begin{array}{c}
\dot{X}(s) \cos \theta \\
\dot{Y}(s) \\
\dot{X}(s) \sin \theta
\end{array}\right] \text { and } \frac{\partial \tilde{\mathbf{S}}_{\mathrm{r}}}{\partial \theta}=\left[\begin{array}{c}
-X(s) \sin \theta \\
0 \\
X(s) \cos \theta
\end{array}\right]
$$


are independent since $\dot{X}(s)$ and $\dot{Y}(s)$ are never simultaneously zero and $X(s)>0$ for all $s$. Hence $\tilde{\mathbf{S}}_{\mathrm{r}}$ is immersed and has a well-defined tangent plane at each point, with the normal given by

$$
\mathbf{n}(s, \theta)=\frac{\partial \tilde{\mathbf{S}}_{\mathrm{r}}}{\partial s} \times \frac{\partial \tilde{\mathbf{S}}_{\mathrm{r}}}{\partial \theta}=\left[\begin{array}{c}
X(s) \dot{Y}(s) \cos \theta \\
-X(s) \dot{X}(s) \\
X(s) \dot{Y}(s) \sin \theta
\end{array}\right]
$$

Through any point $\tilde{\mathbf{S}}_{\mathrm{r}}\left(s_{0}, \theta_{0}\right)$ on the surface, there is a meridian curve which is the curve obtained by rotating $\tilde{\mathbf{C}}_{\mathrm{r}}$ about the $y$-axis by angle $-\theta_{0}$, and a latitude circle which is a circle on the plane $y=Y\left(s_{0}\right)$ and with center on the $y$-axis. Note that the meridian curves and the latitude circles are orthogonal to each other, and they form the principal curves of the surface (see fig. 1). It follows from (3) that the surface normal at $\tilde{\mathbf{S}}_{\mathrm{r}}\left(s_{0}, \theta_{0}\right)$ lies on the plane containing the $y$-axis and the point $\tilde{\mathbf{S}}_{\mathrm{r}}\left(s_{0}, \theta_{0}\right)$, and is normal to the meridian curve through $\tilde{\mathbf{S}}_{\mathrm{r}}\left(s_{0}, \theta_{0}\right)$. By circular symmetry, the surface normals along a latitude circle will all meet at one point on the $y$-axis.

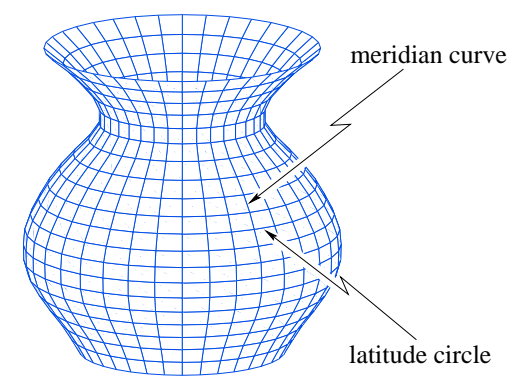

Figure 1: The meridian curves and latitude circles form the principal curves of the surface of revolution.

Under perspective projection, the image of a surface of revolution will be invariant to a harmonic homology [22, 13], given by

$$
\mathbf{W}=\mathbb{I}_{3}-2 \frac{\mathbf{v}_{x} \mathbf{l}_{\mathrm{s}}^{\mathrm{T}}}{\mathbf{v}_{x}^{\mathrm{T}} \mathbf{l}_{\mathrm{s}}}
$$

where $l_{\mathrm{s}}$ is the image of the revolution axis and $\mathbf{v}_{x}$ is the vanishing point corresponding to the normal direction of the plane that contains the camera center and the revolution axis. Note that $\mathbf{W}$ has four degrees of freedom, and that $\mathbf{v}_{x}$ and $\mathbf{l}_{\mathrm{s}}$ are related by [18]

$$
\mathbf{v}_{x}=\mathbf{K K}^{\mathrm{T}} \mathbf{1}_{\mathrm{s}},
$$

where $\mathbf{K}$ is the $3 \times 3$ calibration matrix of the camera. When the camera is pointing directly towards the revolution axis, $\mathbf{v}_{x}$ will be at infinity and the harmonic homology will reduce to a skew symmetry, which has only three degrees of freedom. If the camera also has zero skew and unit aspect ratio, the harmonic homology will then become a bilateral symmetry. The vanishing point will now be both at infinity and has a direction orthogonal to $1_{\mathrm{s}}$, leaving only two degrees of freedom. 


\section{Reconstruction from a Single View}

Consider a surface of revolution $\tilde{\mathbf{S}}_{\mathrm{r}}$ whose revolution axis coincides with the $y$-axis, and a pin-hole camera $\hat{\mathbf{P}}=\left[\begin{array}{ll}\mathbb{I}_{3} & \mathbf{t}\end{array}\right]$ where $\mathbf{t}=\left[\begin{array}{lll}0 & 0 & d_{z}\end{array}\right]^{\mathrm{T}}$ and $d_{z}>0$. Let the contour generator be parameterized by $s$ as

$$
\begin{aligned}
\tilde{\Gamma}(s) & =\tilde{\mathbf{c}}+\lambda(s) \mathbf{p}(s), \text { where } \\
\mathbf{p}(s) \cdot \mathbf{n}(s) & =0 .
\end{aligned}
$$

In (6), $\tilde{\mathbf{c}}$ indicates the camera center at $\left[\begin{array}{lll}0 & 0 & -d_{z}\end{array}\right]^{\mathrm{T}}, \mathbf{p}(s)$ is the viewing vector from $\tilde{\mathbf{c}}$ to the focal plane at unit distance for the point $\tilde{\Gamma}(s)$, and $\lambda(s)$ is the depth of the point $\tilde{\Gamma}(s)$ from $\tilde{\mathbf{c}}$ along the $z$ direction. Note that $\mathbf{p}(s)$ has the form $[x(s) y(s) 1]^{\mathrm{T}}$, where $(x(s), y(s))$ is a point in the silhouette. The tangency constraint is expressed in (7), where $\mathbf{n}(s)$ is the unit surface normal at $\tilde{\Gamma}(s)$ and can be determined up to a sign by [4]

$$
\mathbf{n}(s)=\frac{\mathbf{p}(s) \times \frac{d \mathbf{p}(s)}{d s}}{\left|\mathbf{p}(s) \times \frac{d \mathbf{p}(s)}{d s}\right|}=\frac{1}{\alpha_{\mathrm{n}}(s)}\left[\begin{array}{c}
-\dot{y}(s) \\
\dot{x}(s) \\
x(s) \dot{y}(s)-\dot{x}(s) y(s)
\end{array}\right]
$$

where $\alpha_{\mathrm{n}}(s)=\left|\mathbf{p}(s) \times \frac{d \mathbf{p}(s)}{d s}\right|$. In Section 2, it has been shown that the surface normal $\mathbf{n}(s)$ will lie on the plane containing the $y$-axis and the point $\tilde{\Gamma}(s)$. This coplanarity constraint can be expressed as

$$
\mathbf{n}(s)^{\mathrm{T}}\left[\mathbf{n}_{y}\right]_{\times} \tilde{\Gamma}(s)=0,
$$

where $\mathbf{n}_{y}=\left[\begin{array}{lll}0 & 1 & 0\end{array}\right]^{\mathrm{T}}$. Let $\mathbf{n}(s)=\left[n_{1}(s) n_{2}(s) n_{3}(s)\right]^{\mathrm{T}}$ and expanding (9) gives

$$
\lambda(s)=\frac{d_{z} n_{1}(s)}{n_{1}(s)-n_{3}(s) x(s)} .
$$

Hence, the contour generator can be recovered from the silhouette and, in homogeneous coordinates, is given by

$$
\Gamma(s)=\left[\begin{array}{c}
\tilde{\mathbf{c}}+\lambda(s) \mathbf{p}(s) \\
1
\end{array}\right]=\left[\begin{array}{c}
d_{z} \dot{y}(s) x(s) \\
d_{z} \dot{y}(s) y(s) \\
d_{z} \alpha_{\Gamma}(s) \\
\dot{y}(s)-\alpha_{\Gamma}(s)
\end{array}\right]
$$

where $\alpha_{\Gamma}(s)=(\dot{x}(s) y(s)-x(s) \dot{y}(s)) x(s)$. Since the distance $d_{z}$ cannot be recovered from the image, the reconstruction is determined only up to a similarity transformation. The surface of revolution can then be obtained by rotating the contour generator about the $y$-axis, and is given by

$$
\tilde{\mathbf{S}}_{\mathrm{r}}(s, \theta)=\left[\begin{array}{c}
X(s) \cos \theta \\
Y(s) \\
X(s) \sin \theta
\end{array}\right]
$$

where $X(s)=\sqrt{(\lambda(s) x(s))^{2}+\left(\lambda(s)-d_{z}\right)^{2}}$ and $Y(s)=\lambda(s) y(s)$.

Now consider an arbitrary pin-hole camera $\mathbf{P}$ by introducing the intrinsic parameters represented by the camera calibration matrix $\mathbf{K}$ to $\hat{\mathbf{P}}$, and by applying the rotation $\mathbf{R}$ to 
$\hat{\mathbf{P}}$ about its optical center. Hence $\mathbf{P}=\mathbf{K R}\left[\mathbb{I}_{3} \mathbf{t}\right]$ or $\mathbf{P}=\mathbf{H} \hat{\mathbf{P}}$, where $\mathbf{H}=\mathbf{K R}$. From the discussions presented in Section 2, the resulting silhouette of $\tilde{\mathbf{S}}_{\mathrm{r}}$ will be invariant to a harmonic homology $\mathbf{W}$. Given $\mathbf{K}$ and $\mathbf{W}$, it is possible to rectify the image by a planar homography $\mathbf{H}_{\mathrm{r}}$ so that the silhouette becomes bilaterally symmetric about the line $\hat{\mathbf{l}}_{\mathrm{s}}=\left[\begin{array}{lll}1 & 0 & 0\end{array}\right]^{\mathrm{T}}$ (i.e. the $y$-axis). This corresponds to normalizing the camera by $\mathbf{K}^{-1}$ and rotating the normalized camera until the revolution axis of $\tilde{\mathbf{S}}_{\mathrm{r}}$ lies on the $y-z$ plane of the camera coordinate system. Note that $\mathbf{H}_{\mathrm{r}}$ is not unique, as any homography $\mathbf{H}_{\mathrm{r}}^{\prime}$, given by $\mathbf{H}_{\mathrm{r}}^{\prime}=\mathbf{R}_{x}(\psi) \mathbf{H}_{\mathrm{r}}$ where $\mathbf{R}_{x}(\psi)$ is a rotation about the $x$-axis by an angle $\psi$, will yield a silhouette which will be bilaterally symmetric about $\hat{\mathbf{l}}_{\mathrm{s}}$. There exists $\psi_{0}$ such that $\mathbf{R}_{x}\left(\psi_{0}\right) \mathbf{H}_{\mathrm{r}} \mathbf{P}=\hat{\mathbf{P}}$ and the surface of revolution can be reconstructed from the rectified image using the algorithm presented above. In general, $\psi_{0}$ cannot be recovered from a single image and hence there will be a 2-parameter family of solutions for the contour generator, given by

$$
\Gamma^{\psi}(s)=\left[\begin{array}{c}
d_{z} \dot{y}(s) x(s) \\
d_{z} \dot{y}(s)(y(s) \cos \psi-\sin \psi) \\
d_{z} \alpha_{\Gamma}^{\psi}(s) \\
\dot{y}(s)(y(s) \sin \psi+\cos \psi)-\alpha_{\Gamma}^{\psi}(s)
\end{array}\right]
$$

where $\alpha_{\Gamma}^{\psi}(s)=\{(\dot{x}(s) y(s)-x(s) \dot{y}(s)) \cos \psi-\dot{x}(s) \sin \psi\} x(s)$. The 2-parameter family of surfaces of revolution $\tilde{\mathbf{S}}_{\mathrm{r}}^{\psi}$ can be obtained by rotating $\Gamma^{\psi}$ about the $y$-axis. Note that the ambiguities in the reconstruction correspond to (1) the unknown distance of the surface from the camera and (2) the ambiguity of the orientation of the revolution axis on the $y-z$ plane of the camera coordinate system. It is shown in the Appendix that such ambiguities in the reconstruction cannot be described by a projective transformation. If the image of a latitude circle (e.g. an "angular ridge") in the surface of revolution can be located, the orientation of the revolution axis relative to the $y$-axis of the camera coordinate system can be estimated [5], which removes one degree of freedom in the ambiguities of the reconstruction. Further, if the radius of such a latitude circle is also known, then all the ambiguities in the reconstruction can be resolved.

\section{Algorithm and Implementation}

\subsection{Estimation of the Harmonic Homology}

The silhouette $\rho$ of a surface of revolution is first extracted from the image by applying a Canny edge detector [3], and the harmonic homology $\mathbf{W}$ that maps each side of $\rho$ to its symmetric counterpart is then estimated by minimizing the geometric distances between the original silhouette $\rho$ and its transformed version $\rho^{\prime}=\mathbf{W} \rho$. This can be done by sampling $N$ evenly spaced points $\mathbf{x}_{i}$ along $\rho$ and optimizing the cost function

$$
\operatorname{Cost}\left(\mathbf{v}_{x}, \mathbf{l}_{\mathrm{s}}\right)=\sum_{i=1}^{N} \operatorname{dist}\left(\mathbf{W}\left(\mathbf{v}_{x}, \mathbf{l}_{\mathrm{s}}\right) \mathbf{x}_{i}, \rho\right)^{2},
$$

where $\operatorname{dist}\left(\mathbf{W}\left(\mathbf{v}_{x}, \mathbf{l}_{\mathrm{s}}\right) \mathbf{x}_{i}, \rho\right)$ is the orthogonal distance from the transformed sample point $\mathbf{x}_{i}^{\prime}=\mathbf{W}\left(\mathbf{v}_{x}, \mathbf{l}_{\mathrm{s}}\right) \mathbf{x}_{i}$ to the original silhouette $\rho$. 


\subsection{Image Rectification}

After the estimation of the harmonic homology $\mathbf{W}$, the image can be rectified so that the silhouette becomes bilaterally symmetric about the line $\mathbf{l}=\left[\begin{array}{lll}1 & 0 & 0\end{array}\right]^{\mathrm{T}}$. Such a rectified image resembles an image that would have been observed by a normalized camera when the axis of the surface of revolution lies on the $y$ - $z$ plane of the camera coordinate system.

By assuming that the principal point is located at the image center and that the camera has unit aspect ratio, the focal length can be computed from $\mathbf{v}_{x}$ and $\mathbf{l}_{\mathrm{s}}$ using (5). The image can then be normalized by $\mathbf{K}^{-1}$ to remove the effects of the intrinsic parameters of the camera. The axis $\mathbf{l}_{\mathrm{s}}$ of $\mathbf{W}$, and hence the image of the revolution axis, is transformed to $\mathbf{l}_{\mathrm{s}}^{\mathrm{n}}=\mathbf{K}^{\mathrm{T}} \mathbf{l}_{\mathrm{s}}$.

The normalized image is then transformed by a rotation matrix $\mathbf{R}_{\mathrm{b}}$ that brings $\mathbf{x}_{0}^{\mathrm{p}}$, the orthogonal projection of the principal point $\mathbf{x}_{0}=\left[\begin{array}{lll}0 & 0 & 1\end{array}\right]^{\mathrm{T}}$ on the axis $\mathbf{l}_{\mathrm{s}}^{\mathrm{n}}$, to $\mathbf{x}_{0}$. This corresponds to rotating the normalized camera until it points directly towards the axis of the surface of revolution, and the resulting silhouette $\rho^{\mathrm{b}}=\mathbf{R}_{\mathrm{b}} \mathbf{K}^{-1} \rho$ will then be bilaterally symmetric about the image of the revolution axis, given by $\mathbf{l}_{\mathrm{s}}^{\mathrm{b}}=\mathbf{R}_{\mathrm{b}}^{-\mathrm{T}} \mathbf{l}_{\mathrm{s}}^{\mathrm{n}}=$ $\left[\cos \theta^{\mathrm{b}} \sin \theta^{\mathrm{b}} 0\right]^{\mathrm{T}}$.

The resulting image is then rotated about the point $\mathbf{x}_{0}$ until the axis of symmetry aligns with the $y$-axis, and the transformation is given by $\mathbf{R}_{\mathrm{a}}$ which is a rotation about the $z$-axis by an angle $-\theta^{\mathrm{b}}$. This corresponds to rotating the normalized camera, which is now pointing directly towards the axis of the surface of revolution, about its $z$-axis until the axis of the surface of revolution lies on the $y-z$ plane. The resulting silhouette $\rho^{\mathrm{a}}=$ $\mathbf{R}_{\mathrm{a}} \rho^{\mathrm{b}}$ is now bilaterally symmetric about the line $\mathbf{l}_{\mathrm{s}}^{\mathrm{a}}=\mathbf{R}_{\mathrm{a}}^{-\mathrm{T}} \mathbf{l}_{\mathrm{s}}^{\mathrm{b}}=\left[\begin{array}{lll}1 & 0 & 0\end{array}\right]^{\mathrm{T}}$. The overall transformation for the rectification is given by $\mathbf{H}_{\mathrm{r}}=\mathbf{R}_{\mathrm{a}} \mathbf{R}_{\mathrm{b}} \mathbf{K}^{-1}$, and the rectification process is illustrated in fig. 2 .

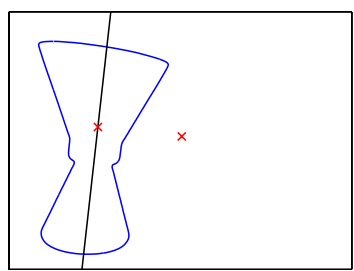

(a)

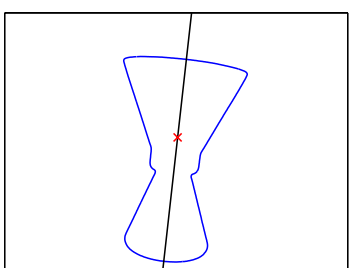

(b)

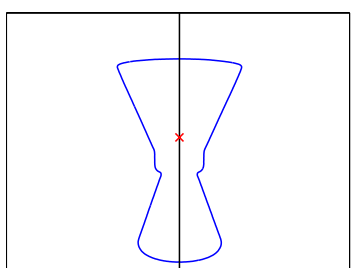

(c)

Figure 2: (a) The harmonic homology associated with the silhouette of the surface of revolution is estimated, which yields the image of the revolution axis. The image is then normalized by $\mathbf{K}^{-1}$, and the orthogonal projection $\mathbf{x}_{0}^{\mathrm{p}}$ of the point $\mathbf{x}_{0}=\left[\begin{array}{lll}0 & 0 & 1\end{array}\right]^{\mathrm{T}}$ on the image of the revolution axis is located. (b) The image is transformed by the homography $\mathbf{R}_{\mathrm{b}}$ so that the point $\mathbf{x}_{0}$ lies on the image of the revolution axis and the silhouette becomes bilaterally symmetric about the image of the revolution axis. (c) Finally, the image is rotated about the point $\mathbf{x}_{0}$ until the image of the revolution axis aligns with the $y$-axis.

\subsection{Depth Recovery}

Since the rectified silhouette $\rho^{\mathrm{a}}$ is bilaterally symmetric about the $y$-axis, only one side of $\rho^{\mathrm{a}}$ needs to be considered during the reconstruction of the surface of revolution. Points 
are first sampled from one side of $\rho^{\text {a }}$ and the tangent vector (i.e. $\dot{x}(s)$ and $\dot{y}(s)$ ) at each sample point is estimated by fitting a polynomial to the neighboring points in the rectified silhouette. The surface normal associated with each sample point is then computed using (8). Finally, the depth of each sample point is recovered using (10), and the contour generator and the surface of revolution follow. For $\psi \neq 0$, the viewing vector $\mathbf{p}(s)$ and the associated surface normal $\mathbf{n}(s)$ at each sample point are first transformed by $\mathbf{R}_{x}(\psi)$. The transformed viewing vector is then normalized so that its $3^{r d}$ coefficient becomes 1 , and (10) can then be used to recover the depth of the sample point.

\section{Experiments and Results}

Fig. 3 shows the reconstruction of a candle holder. The rectification of the silhouette (see fig. 3(b)) was done using the algorithm described in Section 4. An ellipse was fitted to the bottom of the rectified silhouette for computing the orientation of the revolution axis. The radius of the topmost circle and the height of the candle holder, measured manually using a ruler with a resolution of $1 \mathrm{~mm}$, were $5.7 \mathrm{~cm}$ and $17.1 \mathrm{~cm}$ respectively. The ratio of the radius of the topmost circle to the height of the reconstructed candle holder (see fig. 3(c)) was 0.3360 . This ratio agreed with the ground truth value $(5.7 / 17.1=0.3333)$ and had a relative error of $0.81 \%$ only. Another example is given in fig. 4 , which shows the reconstruction of a bowl. The radius of the topmost circle and the height of the bowl were $6.4 \mathrm{~cm}$ and $6.2 \mathrm{~cm}$ respectively. The ratio of the radius of the topmost circle to the height of the reconstructed bowl (see fig. 4(c)) was 1.0474. This ratio was close to the ground truth value $(6.4 / 6.2=1.0323)$ and had a relative error of $1.46 \%$.

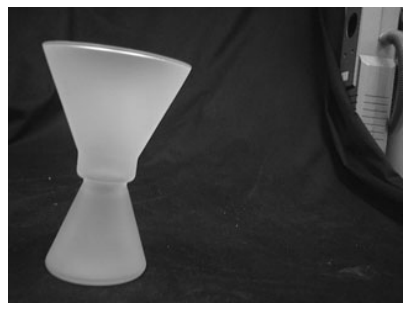

(a)

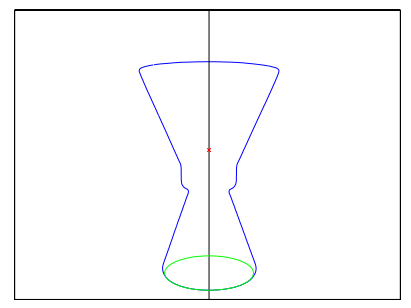

(b)

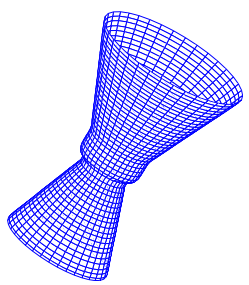

(c)

Figure 3: (a) Image of a candle holder. (b) Rectified silhouette of the candle holder which exhibits bilateral symmetry. (c) Reconstructed model of the candle holder.

\section{Conclusions}

By exploiting the coplanarity constraint between the revolution axis and the surface normal, a simple technique for recovering the $3 \mathrm{D}$ shape of a surface of revolution from a single view has been developed. The technique presented here assumes perspective projection and uses information from the silhouette only. The invariant properties of the surface of revolution and its silhouette have been used to calibrate the focal length of the camera, and to rectify the image so that the silhouette becomes bilaterally symmetric 


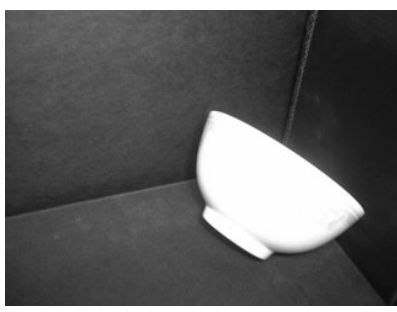

(a)

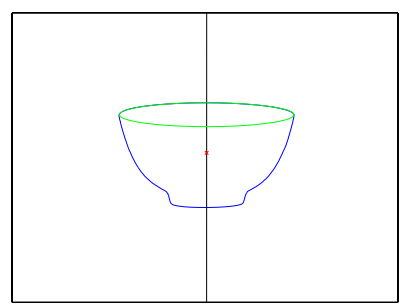

(b)

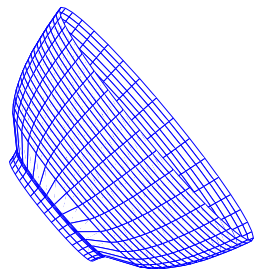

(c)

Figure 4: (a) Image of a bowl. (b) Rectified silhouette of the bowl which exhibits bilateral symmetry. (c) Reconstructed model of the bowl.

about the $y$-axis. This simplifies the analysis of the general camera configuration case to one in which the revolution axis lies on the $y-z$ plane of the camera coordinate system. If the image of a latitude circle in the surface of revolution can be located, the orientation of the revolution axis relative to the $y$-axis of the camera coordinate system can be estimated, which removes one degree of freedom in the ambiguities of the reconstruction. The remaining degree of freedom (i.e. scale) can be resolved by knowing the radius of the latitude circle located.

\section{Appendix: Analysis of the Reconstruction Ambiguities}

A projective transformation that maps a surface of revolution to another surface of revolution, both with the $y$-axis as their revolution axes, has the following generic form

$$
\mathbf{H}_{\mathrm{SOR}}=\left[\begin{array}{cccc}
\cos \varrho & 0 & \sin \varrho & 0 \\
0 & h_{1} & 0 & h_{2} \\
\mp \sin \varrho & 0 & \pm \cos \varrho & 0 \\
0 & h_{3} & 0 & h_{4}
\end{array}\right] \text { where }\left|\begin{array}{ll}
h_{1} & h_{2} \\
h_{3} & h_{4}
\end{array}\right| \neq 0
$$

A detailed derivation for $\mathbf{H}_{\mathrm{SOR}}$ can be found in [18]. Assuming that the ambiguity in the reconstruction resulting from the unknown orientation of the revolution axis can be described by $\mathbf{H}_{\mathrm{SOR}}$, then both $\mathbf{H}_{\mathrm{SOR}}$ and the transformation induced by $\mathbf{R}_{x}(\psi)$ will map a latitude circle in $\tilde{\mathbf{S}}_{\mathrm{r}}$ to the same latitude circle in $\tilde{\mathbf{S}}_{\mathrm{r}}^{\psi}$, as a latitude circle is by itself a surface of revolution in the limiting case. Hence, if the ambiguity is projective, there exists $\varrho$ for each $y(s)$ such that $\Gamma^{\psi}(s)=\mathbf{H}_{\mathrm{SOR}} \Gamma(s)$. In Cartesian coordinates, the projective transformation $\Gamma^{\psi}(s)=\mathbf{H}_{\mathrm{SOR}} \Gamma(s)$, with $d_{z}=1$, is given by the set of equations

$$
\begin{aligned}
\frac{\dot{y}(s) x(s)}{\dot{y}(s)(y(s) \sin \psi+\cos \psi)-\alpha_{\Gamma}^{\psi}(s)} & =\frac{\dot{y}(s) x(s) \cos \varrho+\alpha_{\Gamma}(s) \sin \varrho}{h_{3} \dot{y}(s) y(s)+h_{4}\left(\dot{y}(s)-\alpha_{\Gamma}(s)\right)}, \\
\frac{\dot{y}(s)(y(s) \cos \psi-\sin \psi)}{\dot{y}(s)(y(s) \sin \psi+\cos \psi)-\alpha_{\Gamma}^{\psi}(s)} & =\frac{h_{1} \dot{y}(s) y(s)+h_{2}\left(\dot{y}(s)-\alpha_{\Gamma}(s)\right)}{h_{3} \dot{y}(s) y(s)+h_{4}\left(\dot{y}(s)-\alpha_{\Gamma}(s)\right)} \\
\frac{\alpha_{\Gamma}^{\psi}(s)}{\dot{y}(s)(y(s) \sin \psi+\cos \psi)-\alpha_{\Gamma}^{\psi}(s)} & =\frac{\mp \dot{y}(s) x(s) \sin \varrho \pm \alpha_{\Gamma}(s) \cos \varrho}{h_{3} \dot{y}(s) y(s)+h_{4}\left(\dot{y}(s)-\alpha_{\Gamma}(s)\right)} .
\end{aligned}
$$




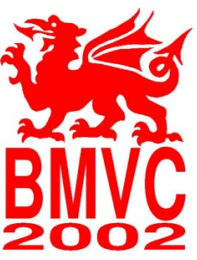

Rearranging (A-3) gives

$$
\begin{aligned}
0= & h_{2} \cos \psi x(s)^{4} \dot{y}(s)^{2}+h_{2} \sin \psi x(s)^{3} \dot{x}(s) \dot{y}(s)- \\
& 2 h_{2} \cos \psi x(s)^{3} \dot{x}(s) y(s) \dot{y}(s)+ \\
& \left(h_{1} \cos \psi+h_{2} \sin \psi-h_{4} \cos \psi\right) x(s)^{2} y(s) \dot{y}(s)^{2}+ \\
& \left(2 h_{2} \cos \psi+h_{4} \sin \psi\right) x(s)^{2} \dot{y}(s)^{2}+ \\
& h_{2} \cos \psi x(s)^{2} \dot{x}(s)^{2} y(s)^{2}-h_{2} \sin \psi x(s)^{2} \dot{x}(s)^{2} y(s)+ \\
& \left(h_{4} \cos \psi-h_{1} \cos \psi-h_{2} \sin \psi\right) x(s) \dot{x}(s) y(s)^{2} \dot{y}(s)+ \\
& \left(h_{1} \sin \psi-2 h_{2} \cos \psi-h_{4} \sin \psi\right) x(s) \dot{x}(s) y(s) \dot{y}(s)+ \\
& h_{2} \sin \psi x(s) \dot{x}(s) \dot{y}(s)+\left(h_{1} \sin \psi-h_{3} \cos \psi\right) y(s)^{2} \dot{y}(s)^{2}+ \\
& \left(h_{1} \cos \psi+h_{2} \sin \psi+h_{3} \sin \psi-h_{4} \cos \psi\right) y(s) \dot{y}(s)^{2}+ \\
& \left(h_{2} \cos \psi+h_{4} \sin \psi\right) \dot{y}(s)^{2},
\end{aligned}
$$

which holds for all values of $x(s), \dot{x}(s), y(s), \dot{y}(s)$ and $\psi$. Equation (A-5) yields the following 8 constraints

$$
\begin{aligned}
h_{2} \cos \psi & =0, \\
h_{2} \sin \psi & =0, \\
h_{1} \cos \psi+h_{2} \sin \psi-h_{4} \cos \psi & =0, \\
2 h_{2} \cos \psi+h_{4} \sin \psi & =0, \\
h_{1} \sin \psi-2 h_{2} \cos \psi-h_{4} \sin \psi & =0, \\
h_{1} \sin \psi-h_{3} \cos \psi & =0, \\
h_{1} \cos \psi+h_{2} \sin \psi+h_{3} \sin \psi-h_{4} \cos \psi & =0, \text { and } \\
h_{2} \cos \psi+h_{4} \sin \psi & =0 .
\end{aligned}
$$

Solving equations (A-6)-(A-13) gives

$$
h_{1}=h_{2}=h_{3}=h_{4}=0,
$$

which makes $\mathbf{H}_{\mathrm{SOR}}$ singular. As a result, the ambiguity in the reconstruction cannot be described by a projective transformation.

\section{References}

[1] S. T. Barnard and M. A. Fischler. Computational stereo. ACM Computing Surveys, 14(4):553-572, December 1982

[2] T. O. Binford. Visual perception by computer. In Proc. IEEE Conf. Systems and Control, Miami, FL, December 1971.

[3] J. Canny. A computational approach to edge detection. IEEE Trans. on Pattern Analysis and Machine Intelligence, 8(6):679-698, November 1986.

[4] R. Cipolla and A. Blake. Surface shape from the deformation of apparent contours. Int. Journal of Computer Vision, 9(2):83-112, November 1992.

[5] M. Dhome, J. T. La Preste, G. Rives, and M. Richetin. Spatial localization of modelled objects of revolution in monocular perspective vision. In O. Faugeras, editor, Proc. 1st European Conf. on Computer Vision, volume 427 of Lecture Notes in Computer Science, pages 475-485, Antibes, France, April 1990. Springer-Verlag. 
[6] A. D. Gross and T. E. Boult. Recovery of SHGCs from a single intensity view. IEEE Trans. on Pattern Analysis and Machine Intelligence, 18(2):161-180, February 1996.

[7] R. Horaud and M. Brady. On the geometric interpretation of image contours. Artificial Intelligence, 37:333-353, December 1988.

[8] J. J. Koenderink and A. J. van Doorn. Geometry of binocular vision and a model for stereopsis. Biological Cybernetics, 21:29-35, 1976.

[9] J. M. Lavest, R. Glachet, M. Dhome, and J. T. La Preste. Modelling solids of revolution by monocular vision. In Proc. Conf. Computer Vision and Pattern Recognition, pages 690-691, Lahaina, Maui, HI, June 1991.

[10] J. Liu, J. L. Mundy, D. A. Forsyth, A. Zisserman, and C. A. Rothwell. Efficient recognition of rotationally symmetric surface and straight homogeneous generalized cylinders. In Proc. Conf. Computer Vision and Pattern Recognition, pages 123-129, New York, NY, June 1993.

[11] H. C. Longuet-Higgins and K. Prazdny. The interpretation of a moving retinal image. Proc. Royal Soc. London B, 208:385-397, 1980.

[12] J. Malik and R. Rosenholtz. Computing local surface orientation and shape from texture for curved surfaces. Int. Journal of Computer Vision, 23(2):149-168, June 1997.

[13] P. R. S. Mendonça, K.-Y. K. Wong, and R. Cipolla. Epipolar geometry from profiles under circular motion. IEEE Trans. on Pattern Analysis and Machine Intelligence, 23(6):604-616, June 2001.

[14] J. Ponce, D. M. Chelberg, and W. B. Mann. Invariant properties of straight homogeneous generalized cylinders and their contours. IEEE Trans. on Pattern Analysis and Machine Intelligence, 11(9):951-966, September 1989.

[15] H. Sato and T. O. Binford. Finding and recovering SHGC objects in an edge image. Computer Vision, Graphics and Image Processing, 57(3):346-358, May 1993.

[16] S. Ullman. The Interpretation of Visual Motion. MIT Press, Cambridge, MA, 1979.

[17] F. Ulupinar and R. Nevatia. Shape from contour: Straight homogeneous generalized cylinders and constant cross-section generalized cylinders. IEEE Trans. on Pattern Analysis and Machine Intelligence, 17(2):120-135, February 1995.

[18] K.-Y. K. Wong. Structure and Motion from Silhouettes. PhD thesis, Department of Engineering, University of Cambridge, 2001

[19] M. Zerroug and R. Nevatia. Three-dimensional descriptions based on the analysis of the invariant and quasi-invariant properties of some curved-axis generalized cylinders. IEEE Trans. on Pattern Analysis and Machine Intelligence, 18(3):237-253, March 1996.

[20] M. Zerroug and R. Nevatia. Volumetric descriptions from a single intensity image. Int. Journal of Computer Vision, 20(1/2):11-42, 1996.

[21] R. Zhang, P. S. Tsai, J. E. Cryer, and M. Shah. Shape from shading: A survey. IEEE Trans. on Pattern Analysis and Machine Intelligence, 21(8):690-706, August 1999.

[22] A. Zisserman, J. L. Mundy, D. A. Forsyth, J. Liu, N. Pillow, C. Rothwell, and S. Utcke. Class-based grouping in perspective images. In Proc. 5th Int. Conf. on Computer Vision, pages 183-188, Cambridge, MA, USA, June 1995 